\title{
EL CONTROL DE CALIDAD Y OPORTUNIDAD DE LOS INFORMES DE AUDITORÍA
}

\author{
Dr.'JULIO V. FLORES KONJA \\ CPCALBERTO MONTES FARRO, Mg. ADRIÁNA. FLORES KONJA, LiC. ANA MARÍA \\ GUTIÉRREZ HUBY, CPC JORGE GAVELÁN IZAGUIRRE, Ing. EFREN MICHUE SALGUEDO
}

\section{ANTECEDENTES HISTÓRICOS DEL CONTROL DE CALIDAD}

Antes de dar inicio al desarrollo de los antecedentes es interesante efectuar una descripción de lo que a nuestro concepto significa una auditoría de calidad.

Una de la definiciones clásicas de la auditoría la contempla como el "examen de unos estados financieros realizado por profesionales competentes e independientes, mediante la aplicación de procedimientos con unas normas con el objeto de emitir una opinión sobre la razonabilidad con la cual dichos estados financieros presentan la situación financiero-patrimonial a una fecha dada (balance de situación), el resultado de las operaciones de un ejercicio (estado de pérdidas y ganancias), etc., todo ello con respecto a unos principios de contabilidad generalmente aceptados".

\subsection{Edad Antigua}

En las antiguas civilizaciones se aprecia permanentemente la preocupación de los hombres por aplicar conceptos y prácticas bien realizadas en el desarrollo de sus actividades. Sin embargo, antes de la existencia de legisladores como Salomón, surgió la necesidad de un método, de un sistema para gobernar y administrar.

Muchos de los legisladores antiguos dieron a sus servidores de confianza la autoridad suficiente para actuar en su lugar, controlando la calidad de las decisiones emanadas que permitieran la aplicación de medidas justas.

Se han hallado documentos de la civilización Sumeria con una antigüedad de cinco mil años que evidencian prácticas de controles de calidad administrativos. Así notamos que el tema se complicaba en caso de defunción del sacerdote, había que establecer un sistema de control de controles o auditoría que calificaban la calidad de la gestión del funcionario y mediante la cual se podía asegurar la continuidad de las transaciones comerciales trasmitidas a su sucesor. Es muy probable que las necesidades de control que se ejercía en esta antigua civilización hayan conducido a la invención de su escritura.

En los Egipcios, la demostración más obvia de la destreza en la calidad de sus controles la observamos cuando, por ejemplo, el autor Alberto Arebalo nos narra en uno de los párrafos de su libro Contabilidad General que los monarcas en sus guerras iban acompañados de escribas, quienes tomaban nota de las cosas que componían el botín, de los campos cultivados y de su producción y de lo recogido en el camino.

El autor Reyner afirma que los escribas empezaron a llevar dos contabilidades: una 
muy complicada y oscura, que presentaban a sus superiores para hacerles creer que se trataba de una tarea laboriosa y complicada y mantener el monopolio de la misma, y la otra más sencilla destinada a su uso particular. Pudieron así substraer a los nuevos dominadores cantidades de productos que luego en forma amigable y secreta repartían entre ellos.

En Babilonia, el Rey Hammurabi fue el creador de un conjunto de leyes, llamado el Código de Hammurabi. En este documento se hacía notar especialmente que si el trabajo no se llevaba a cabo adecuadamente sería el supervisor de los hombres y no los hombres, quien sería castigado. Estas medidas de control demuestran una férrea búsqueda por la calidad de los controles.

Otro rey babilonio, Nabucodonosor, volvió a preocuparse seriamente por los controles. Durante su gobierno se centró en un exquisito control de la calidad de la supervisión en los talleres textiles, en donde se utilizaba el método de la coloración para controlar la entrada del hilo de lino a fabricar cada semana. El mismo tipo de sistema era provisto en los graneros donde la entrada de las tinajas se marcaba con diferentes colores cada año.

\subsection{Oriente - La China}

La constitución de Chow, probablemente escrita alrededor de 1100 a.C. proporcionó descripciones específicas de trabajo para todos los servidores públicos del emperador, desde el Primer Ministro hasta el más humilde sirviente de la casa. Los trabajos, deberes y los límites que se esperan de cada persona se señalan en chino simple, de uso diario. Así cuando se mencionaban los poderes que debía manejar el Primer Ministro decía: Ocho cosas retiene el rey para ayudarlo a manejar a sus numerosos oficiales: la primera, el rango que controla su distinción; la segunda, el emolumento que controla su riqueza; la tercera, el favor que controla su fortuna; la cuarta, la estipulación que controla su conducta; la quinta, su cuidado que controla sus mercedes; la sexta, la confiscación que controla su desamparo; la séptima, la remoción que controla sus fallos y la octava, la muerte que controla sus abusos.

Otro filósofo chino importante de mencionar es Sun Tzu del cual encontramos escritos de alrededor de 500 años a. C. El Arte de Guerra es el más viejo tratado militar en el mundo y en él previno "cuando el general es débil y sin autoridad, cuando sus órdenes no son claras ni precisas; donde no hay deberes fijos asignados a hombres y oficiales; una extrema desorganización será el resultado".

Asimismo, los antiguos chinos destacaron la importancia de controlar la selección de aquelllos hombres que desempeñarían funciones públicas. La selección científica de trabajadores por medio de exámenes fue establecida por el gobierno chino alrededor de 120 años a.C.

\subsection{Era Clásica - La Administración Griega}

La Era Clásica centró la atención de la historia nuevamente en el Mediterráneo durante mil años más o menos. Cuestionando toda clase de ideas y conocimientos, los griegos descubrieron los criterios de la investigación para la búsqueda de la calidad e introdujeron la ciencia y la educación en muchas esferas. Uno de los mecanismos que introdujeron para mediatizar el cansancio de sus trabajadores fue el ritmo musical aplicado a través de la flauta y clarinete, lo cual aplicado a los tiempos de trabajo y movimientos tipo, trabajando en armonía con la música. 
A pesar de la baja clasificación en popularidad, la población de mercaderes prosperó en Grecia. La banca se estableció adecuadamente entre los clientes individuales. Dentro de las paredes el templo encontramos un escrito en el cual se menciona por ejemplo: "Deje que otro dé las excusas, pero Caicus aún paga de noche en moneda extranjera a aquellos que la desean".

Veamos brevemente las ideas de tres filósofos, para mayor conocimiento del medio ambiente de los hombres de negocios griegos:

Platón.- En su legislación recomendó un conjunto de leyes y reglamentos bajo el supuesto de que la compra y la venta eran casi sinónimas de mentira y robo. De esta posición solamente se definía una conclusión, de que las leyes deberían eliminar todos los negocios.

Aristóteles.- Para su modo de ver las cosas, el interés representaba dinero que no se sabía de dónde venía (...) que no era pagado en cambio de algo. Discutió cuestiones económicas tales como el cambio, la división de la mano de obra, el dinero, el interés y la usura.

Jenofonte.- Veía la actividad comercial desde un punto de vista más realista. Veía a los negocios como la oportunidad para aumentar los tesoros del estado, y surgió para que se alentara el comercio.

\subsection{La Edad Media en Venecia}

La asociación y la empresa en comandita fueron las dos formas principales de organizar los negocios en el renacimiento italiano. La práctica italiana antigua de pagar a los agentes una participación de las ganancias abrió el camino a la costumbre de pagar un porcentaje fijo de la transacción.
Los elementos de la partida doble fueron usados en el libro mayor de los banqueros en 1340 y los libros de los comerciantes-banqueros florentinos de esa generación contuvieron algunos de los elementos.

El arsenal de Venecia en un intento por alcanzar la calidad de las operaciones llevó una cuenta estricta de monedas, materiales y hombres.

Con el surgimiento de diferentes problemas resultantes del crecimiento del Arsenal, la contabilidad fue la primera en ser reconocida y aceptada como una técnica de control en la administración de dicho negocio. De los libros de cuentas manejados, uno lo guardaba el director del Arsenal, que velaba por la caja en efectivo; y el otro era guardado por el Subjefe del cual el jefe de contadores registraba las partidas en el mayor.

\subsection{Periodo de Medioevo en América del Sur}

Las tremendas distancias y la enorme proporción territorial adquirida por el Imperio Inca plantean el problema de la administración de tan vastos territorios. Surge la pregunta de quiénes tenían la responsabilidad de la marcha del gobierno, y quiénes la ejercían. Aparecen, entonces los Quipukamayoc como responsables de infinitas tareas que consistían en controlar los ingresos del Estado, el correcto almacenamiento de los bienes acumulados, la planificación de las diversas fuerzas de trabajo exigidas a los curacas para las más diversas tareas, además de saber cuánta gente podía ser levada en cada región para la formación de los ejércitos.

Según el cronista Huamán Poma de Ayala a estos funcionarios se les reclutaba de las capas bajas de la población, no 
teniendo referencia histórica de quipukamayocs que hayan pertenecido a la aristocracia.

Como elementos de trabajo, el quipucamayoc tenía las guillas, los nudos para registrar las cantidades y la yupana, especie de ábaco para el cálculo abreviado, habiéndose descifrado a la fecha, la forma de registrar cantidades en los quipus, mas no los significados de los colores de las cuerdas, mientras que de la yupana se desconoce su manejo.

\subsection{La Auditoría en la Colonia}

Durante toda la Colonia, exceptuando los años 1785 a 1790 , se utilizó para el registro de la contabilidad fiscal, el modelo de contabilidad por partida simple.

El modeloque utilizaba el Libro Común de Cargos y Datas, con vigencia desde la llegada de los españoles hasta 1600 fue ampliado en 1799 durante la visita de Don José de Areche, quien dispuso que el Libro Manual se separara en dos: Libro Manual de Cargos y Libro Manual de Datas, los que tuvieron vigencia hasta 1785 y cuyo modelo reformado fue utilizado hasta el fin del Virreynato. Este modelo tuvo vigencia hasta el año 1865 en que por Ley del Congreso Peruano, se oficializó el uso e la contabilidad por partida doble.

En la época de la Colonia existió la auditoría con el nombre de "Reveer las cuentas", y fue una preocupación constante de los gobernadores españoles.

Ambos modelos de revisión de cuentas contaron con una amplia pero bien estructurada normatividad, concordante con lo establecido en los pocos libros de textos, que sobre la materia circulaban en la época de la Colonia.

\subsection{Edad Moderna}

Entre los más importantes eventos encontrados podemos mencionar: el crecimiento de las ciudades, la aplicación del principio de especialización, el uso extendido de la imprenta tipográfica y los principios de la revolución industrial.

Aparte de las implicaciones ya anotadas, la única función que recibió mucha atención bajo este sistema fue la de control de materiales.

La forma más común de control de calidad fue la inspección del producto por el comprador, bajo la ley del derecho común de caveat emptor, que significaba la advertencia de falta de peso, calidad o fuerza debidos.

La era industrial supuso la aparición de un nuevo concepto de "dueño", aquel cuyo único vínculo con su empresa era el capital aportado que se traducía en la distribución de un dividendo al final de cada ejercicio económico.

Su participación en la marcha diaria de las operaciones era mínima, cuando no nula, la misma había sido delegada a los administradores de la empresa. Todo ello generó la necesidad de contar con técnicos contables independientes que garantizaran la bondad de la información suministrada por los administradores y que intervinieran en las liquidaciones de las sociedades que suspendían pagos o quebraban. De esta manera surgió la profesión de "Accountant" o"auditor".

Sin embargo, como ocurre en toda profesión nueva, comenzaron a surgir intrusos que dificultaban la labor de los auténticos profesionales y ponían en tela de juicio la reputación de la profesión. El 
nacimiento de la profesión en el resto de los países europeos tardaría algunos años más.

Posiblemente, como consecuencia de la mayor lentitud en la instauración de la industrialización, la influencia de ésta sobre los intereses del ahorrador privado no se hizo sentir tan pronto. El único vehículo que se intentó para proteger al accionista minoritario fue el consejo de vigilancia, órgano de la propia sociedad que vigilaba la actuación de los consejos de administración y las gerencias.

\subsection{Edad Contemporánea}

La influencia de la legislación y de la mentalidad inglesa en Estados Unidos ha perdurado muchos años después del 4 de julio de 1776, día de su independencia. Es verdad que hasta la consolidación definitiva de la profesión tuvieron que pasar algunos años y no fue sino hasta después de la depresión de 1929 cuando empezaron a dictarse normas legales que hacían obligatoria la auditoría, lo cual no significaba que únicamente la fuerza de la ley provocara el arraigo de la profesión, pues desde las últimas décadas del siglo XIX, quizá por una costumbre iniciada con la dominación inglesa, se había venido requiriendo por un gran número de sociedades, la intervención de auditores que garantizaran la veracidad y corrección de los estados financieros. Fueron precisamente la excelente reputación y el prestigio profesional de los auditores americanos, los factores más importantes que contribuyeron a que la auditoría fuese una base fundamental en el desarrollo económico de las empresas.

Para evitar los criterios heterogéneos de las leyes de distintos estados, en el año 1916 se crea el "American Institute of Certified Public Accountant" que se encarga de expedir los certificados de contador público, previo examen de conocimientos técnicos y cumplimiento de ciertos requisitos.

En 1930, y a solicitud de la bolsa de New York, aún bajo los efectos del crack del año anterior, el Institutodesignó un comité para asesorar a la Bolsa sobre problemas de contabilidad, análisis de balances y el contenido y principios que debían gobernar la preparación de los estados financieros de las empresas que se cotizarían en la Bolsa de New York.

En los años 1932 y 1933 el profesor Amsterdam publicó famosos ensayos sobre la responsabilidad del auditor que decían: "La función general del auditor en la sociedad proviene de la necesidad de ésta de contar con un examen experto e independiente".

Esta función tiene sus raíces en la confianza que la sociedad pone en la efectividad de la auditoría y en la opinión del auditor. Esta confianza es, por tanto, condición para que exista tal función, si se traiciona la confianza, la función al volverse inútil, queda destruida. El contador (auditor) está obligado a realizar su trabajo de tal manera que no traicione las expectativas que despierta en el público lego: y por el contrario, el auditor no debe crear falsas expectativas, debiendo éstas ser justificadas únicamente por el trabajo realizado.

\section{NORMAS SOBRE CONTROL DE CALIDAD DE AUDITORÍA}

En relación a las normas sobre control de calidad de auditoría, en los últimos años, se ha emitido normas que están vigentes y que constituyen las herramientas básicas para el desarrollo e idoneidad de los trabajos de auditoría. Entre estas tenemos las siguientes: 


\subsection{Las Normas Internacionales de Auditoría (NIA)}

Aprobado por el Consejo de la Federación Internacional de Contadores (IFAC) en julio de 1994, en vigencia a partir del $I^{\circ}$ de julio de 1966 , emitida por el Comité Internacional de Práctica de Auditoría (IAPC). El Número de Tema y Título del Documento corresponde al 220 "Control de Calidad para el trabajo de Auditoría".

Esta norma internacional tiene el propósito de establecer normas y proporcionar lineamientos sobre el Control de Calidad, señalando políticas y procedimientos que una firma de auditoría deberá implementar, así como los procedimientos a seguir del trabajo delegado a auxiliares en una auditoría. El cumplimiento de esta norma implica el diseño de políticas y procedimientos de control de calidad que asegure que todas las auditorías son conducidas de acuerdo a Normas Internacionales de Auditoría o con Normas y prácticas nacionales relevantes.

En lo referente al sector público en algunos países, señala que el control de calidad generalmente tiene diferente significado al adoptado en esta NIA. Así, asegurar la calidad es el término aplicado a revisiones de calidad externa.

\subsection{Las Normas Internacionales de Auditoría (NIA)}

Emitida por el Colegio de Contadores Públicos de Lima en el año 1993. Este tema corresponde al número 240 "Control de Calidad del Trabajo de Auditoría" - NIA 7. Constituye la norma que la profesión de contador público debe utilizar para desarrollar la auditoría de los estados financieros en todos los países miembros del IFAC.
El contenido de esta norma sigue los lineamientos del IFAC, desarrollando la Introducción, Controles específicos de calidad, Controles generales de calidad y un apéndice de ejemplos de procedimientos de control general de calidad.

\subsection{Las Normas de Auditoría Gubernamental de la Contraloría de EE.UU - GAO - 1994}

En su última edición, correspondiente a la revisión del "Libro Amarillo" junio de 1994, la GAO, el Contralor de Estados Unidos manifiesta: "Para satisfacer las exigencias de gobiernos cada vez más responsables y a la vez, más sensibles a la relación costo-beneficios esperados, los encargados del diseño de políticas y los gerentes requieren información confiable sobre aspectos financieros y operativos. Por tanto, la seguridad que proporcionan los auditores con respecto a dicha información y a los sistemas que la producen es quizás ahora más importante que nunca. La confianza en los auditores amplía la necesidad de normar para guiar a los auditores y permitir a otros confiar en el trabajo de auditoría".

Estas normas constituyen declaraciones amplias de las responsabilidades del auditor, encontrándose en el Capítulo 3 Normas Generales, la norma Control de Calidad que señala que "Las organizaciones de auditoría que lleven a cabo auditorías deben tener un apropiado sistema de control de calidad". Esta norma mantiene los mismos lineamientos que las anteriores, sin embargo, precisa que las organizaciones que realicen auditorías de acuerdo con estas normas deben someterse, por lo menos cada tres años a una revisión externa del Control de Calidad, la que deberá ser practicada por una organización que sea independiente de ellas. Asimismo, el programa de revisión externa de control de calidad debe estar orientado a determinar 
si se ha establecido un sistema interno de control de calidad que actúa con eficacia y con seguridad razonable del cumplimiento de las normas de auditoría. También señala los requisitos para una revisión externa de control de calidad.

\subsection{Normas de Auditoría Gubernamental de la Contraloria General de la República del Perú}

Las NAGU (Normas de Auditoría Gubernamental se aprobó mediante Resolución de Contraloría $\mathrm{N}^{\circ}$ 162-95 CG, en la codificación 100 Normas Generales, se identifica el Código 1.60 "Control de Calidad". Esta norma ha sido restituida según Resolución de Contraloría N 141-99-CG del 25 de noviembre de 1999, quedando como sigue: "Los órganos conformantes del Sistema Nacional de Control y las Sociedades de Auditoría designadas que ejecutan Auditoría Gubernamental de acuerdo con estas normas, deberán establecer y mantener un adecuado sistema de control de calidad que permita ofrecer seguridad razonable, que la auditoría se ejecuta en concordancia con las Normas de Auditoría Gubernamental, el Manual de Auditoría Gubernamental y las Guías de Auditoría.

Esta norma recientemente aprobada mantiene los lineamientos de las anteriores, precisando los términos "Control de Calidad Interno" y "Control de Calidad Externo" lo cual significa un avance, especialmente, en lo referente al control de calidad externo, aplicable periódicamente a los Órganos de Auditoría Interna y de las Sociedades de Auditoría designadas.

\section{LA AUDITORÍA INTERNA Y EL CONTROL DE LA CALIDAD}

\subsection{Control de Calidad}

En ejercicio de la auditoría implica cumplir con normas profesionales y legales, y particularmente, los objetivos de trabajo previstos y llegar a conclusiones correctas, lo cual hace necesario ejercer un control de la calidad del trabajo del auditor, siendo necesario establecer medidas de control de la labor realizada a través de los socios de la sociedad, supervisores o de un comité creado expresamente para ese fín. Estas medidas constituyen un medio de autocontrol que garantice la calidad del servicio y reduzca el riesgo de error e incluso negligencia, en perjuicio del cliente y de la colectividad en general.

El control de la calidad de la auditoría tiene relación con el tamaño de la firma, si ésta es pequeña es ejercida directamente por el socio (s) sobre un reducido número de personal y pequeña cartera de clientes. En cambio, en una firma grande al haber una mayor cantidad de trabajo y contar con numeroso personal, aumenta el riesgo de incumplimiento de las normas profesionales y legales, escapándose del control de los socios y gerentes, siendo susceptible que en un determinado momento sea revelado, y como tal cuestionado el trabajo, con las implicancias negativas que generan estos hechos.

Las firmas pequeñas de auditoría ejercen una autoadhesión a normas de control de calidad; pero para las firmas grandes, se plantea que el examen del control de la calidad aplicado y expresado en sus procedimientos, papeles de trabajo e informes de auditoría, sea efectuado periódicamente por contadores públicos externos, expresamente contratados, para obtener una seguridad razonable de que la 
firma aplica las normas profesionales y legales que regulan la actividad de auditoría.

A nivel de cada trabajo de auditoría el control de la calidad usualmente es ejercido por quien firma el informe de auditoría, quien no sólo es responsable de su trabajo personal, sino también por la supervisión del responsable del personal subalterno y revisión del trabajo delegado, debiéndose asegurar que todos ellos laboren de acuerdo al nivel profesional que le corresponda.

\subsection{Clasificación de los Controles Internos de Calidad}

Enrique Fowler Newton en su obra "Auditoría Aplicada" clasifica los controles de calidad aplicable a la auditoría en dos grupos:

\section{a) Controles generales}

Está constituido por un conjunto de procedimientos que indirectamente afectan a todas las auditorías, planteado por organizaciones profesionales de dimensión internacional, tales como:

-American Institute of Certified Public Accountants (AICPA).

-International Federation of

Accountants (IFAC).

-Instituto Mexicano de Contadores

Públicos (IMCP).

-Quality Control Standards Committee (QCSC).

Estos controles inciden sobre las cualidades personales del auditor, políticas de aceptación y continuidad de clientes, implantación y comunicación de controles, evidencia de los controles aplicados, inspección de los papeles de auditoría e informes emitidos.

b) Controles sobre auditorías individuales
Independientemente de que se ejerce supervisión del personal y de los papeles de trabajo sobre las auditorías individuales, es recomendable que la firma auditora tenga establecido que antes de la entrega de los informes de auditoría, los papeles de trabajo sean revisados por un socio o gerente que no haya participado en la auditoría.

\subsection{Características del Control en Auditoría}

Es usual que los gobiernos de cada nación a través de las normas legales regulen el ejercicio de la auditoría, ejerciendo permanentemente una supervisión sobre el ejercicio profesional desarrollado, particularmente si estas se realizan en las entidades y empresas públicas.

International Auditing Guideline, en su boletín $\mathrm{N}^{\circ} 7$, menciona las características más representativas del control de calidad de los servicios de auditoría. Entre otros aspectos estas revisiones de control de calidad deben tender a asegurar que:

-Los profesionales se adhieren a los principios de integridad, objetividad, independencia y confiabilidad que regulan su conducta.

-Los profesionales son competentes en su actividad profesional.

-Los diversos trabajos fueron asignados a quienes poseen la capacidad técnica y experiencia necesaria para ejecutarlos;y

-Existió una adecuada dirección y supervisión del trabajo realizado.

Los sistemas de control de calidad establecidos han de tener relación con la naturaleza y alcance del servicio prestado, así como el tiempo y lugar donde se presta el servicio y la relación costo/beneficio del control. 
La aplicación en la labor de auditoría de un control de calidad eficiente y eficaz, genera una actitud innovadora en la dirección de la firma y en la labor de campo, repercute positivamente en el nivel del servicio brindado al cliente, evidenciando el cumplimiento de la responsabilidad asumida, y la apreciación favorable de los clientes sobre la integridad del auditor; y un espíritu positivo para alcanzar la excelencia de la calidad.

\subsection{Auditoría de Calidad a las Organizaciones}

El propósito de la auditoría consiste en determinar si el sistema de calidad implementado por la empresa se ajusta a las normas de calidad existente, constituyéndose en un parámetro de verificación y evaluación a utilizar en el desarrollo de la auditoría, lo que implica evaluar del cliente los documentos y manuales que regulan sus actividades a nivel de las diferentes secciones y departamentos, procedimientos y normas establecidos, lo que debe dar como resultado, concluir si estos son aptos para atender una necesidad específica, caso contrario, se tipificaría como que son de baja o insuficiente calidad.

Para la realización del examen se debe recolectar la evidencia necesaria, analizar los manuales, comprender los procesos en él señalados y responder a preguntas sobre el trabajo como: ¿En qué consiste?, ¿quién lo hace?, ¿dónde, cómo y cuándo se hace?, etc. En el desarrollo de esta fase es útil auxiliarse de narrativas y diagramas de flujo.

En los diferentes niveles de la organización el sentido de la calidad debe estar presente, y formar parte del compromiso del personal, debiendo, los niveles gerenciales, ejercer un liderazgo al respecto, propendiendo a mejorar los procesos a través del conocimiento de las actividades desarrolladas que no se han ajustado a los niveles de calidad, evaluando las acciones correctivas y preventivas adoptadas. El seguimiento del control, gestión y resultado de los procesos, es imprescindible para evaluar los niveles de calidad aplicados.

\subsection{Replanteamiento de la Función del Auditor y el Control de Calidad}

En los últimos años se está imponiendo en el ambiente profesional de la auditoría de que el rol del auditor (interno o externo), derive de ejercer el "control de los controles" y de expresar opinión sobre la razonabilidad de los estados financieros, asumiendo la labor de un asesor de la dirección de la empresa en temas de calidad y riesgo, lo que implica redefinir las áreas de competencia profesional.

Lo enunciado implica desarrollar procedimientos que hagan viable que el auditor (en particular el interno) alcance sus objetivos y metas previstas, en apoyo de la gestión de la empresa, lo que hace necesario que el auditor evalúe la eficiencia operativa de la empresa, identificando las áreas "problema" de la organización, adoptando medidas que mejoren la gestión, poniéndolas al nivel de un mercado muy competitivo.

Si ejercer el control de la calidad de las labores "rutinarias" del auditor implica desarrollar y aplicar una metodología a evaluar la eficiencia y eficacia de su función, sobre el cual todavía no hay consenso en el medio profesional, el asunto se complica ante el replanteamiento de las funciones del auditor, de que ya no sea únicamente "espectador", sino también "actor", que sienta el beneplácito y la angustia de codirigir a la empresa. Pero se mantiene la interrogante: ¿Cómo ejercer el control de la calidad?; la respuesta corresponde en la medida en que la dirección hubiera alcanzado sus objetivos y metas. 


\section{SITUACION Y PROYECCIÓN DEL CONTROL DE CALIDAD Y OPORTUNIDAD DE LOS INFORMES DE AUDITORÍA}

\subsection{Situación y Proyección}

En la actualidad, principalmente a nivel internacional, se manifiesta la necesidad de acentuar la confiabilidad en los informes de audtoría que se emiten, sobre todo en las grandes firmas, por los comentarios negativos de los organismos internacionales de crédito, que señalan que las auditorías no revelaron en su oportunidad situaciones que han dado lugar a estado de crisis financieras empresariales en algunos países y que han comprometido a otros a nivel mundial. Ante esta situación el tratamiento tradicional está en proceso de cambio, cuya proyección mejora el control de la calidad de los informes de auditoría, para lo cual la especialidad de auditoría debe replantear controles que sean de carácter obligatorio a la profesión, a nivel mundial. Corresponde a la IFAC continuar con el liderazgo en este campo de la actividad de la especialidad de auditoría.

\subsection{Controles de Calidad de Auditoria}

De acuerdo a las experiencias y normatividad vigentes, principalmente en la Contraloría de EE.UU.-GAO y de las grandes firmas de auditoría, y en función de la oportunidad se clasifica como sigue:

a) Control de calidad interna de la

Auditoría.

Corresponde a las organizaciones responsables de efectuar la auditoría diseñar un apropiado sistema de control interno que asegure las políticas y procedimientos a seguir en los trabajos delegados al personal de auditoría. En tal sentido, el control de calidad abarca todo el proceso de desarrollo de la auditoría en estricto cumplimiento de las normas de planificación ejecución y emisión del informe, destacando en este proceso la supervisión sobre el trabajo delegado y al culminarlo, se ejecuta el control por los niveles superiores, socios $y / o$ gerentes que confirmen que las políticas y procedimientos establecidos sobre el control de calidad se han aplicado satisfactoriamente, lo cual implica asegurar la calidad de los informes que emite la organización.

Este control es el más generalizado, cumpliéndose con mayor énfasis en las grandes organizaciones de auditoría, en la que participan diversos niveles, en tanto que en las medianas y pequeñas firmas de auditoría el control de la calidad del trabajo de auditoría se efectua básicamente a través de la supervisión.

b) Control de calidad externo de Auditoría.

Comprende a las organizaciones responsables de efectuar la auditoría diseñar un apropiado sistema de control externo que asegure el cumplimiento de las políticas y procedimientos de los programas de revisión externa del control de calidad por personal idóneo e independiente de la organización la misma que debe efectuarse periódicamente, así la Contraloría de Estados Unidos - GAO ha establecido que por lo menos cada tres años se efectúe una revisión externa del control de calidad y en el caso de las grandes firmas de auditoría la periodicidad es variable.

\subsection{Control de Calidad y Costo de la Auditoría}

El control de la calidad de la auditoría, en su mayor parte se ve afectada por los costos, en las actuales circunstancias de mercados de amplia competencia se considera que cumplir con todos los procedimientos y normatividad constituye una desventaja; así en la práctica para el cumplimiento del servicio se adopta el menor costo posible, minimizando los errores y 
gastos innecesarios, de tal suerte que las labores deben hacerse bien, desde su inicio. Un sistema de control de la calidad de la auditoría basado en la supervisión, bien llevado y exigente puede reducir los costos en esta parte, y reservar gastos para un posterior control de calidad externa de auditoría realizada por una firma totalmente independiente.

\section{CONCLUSIONES}

1.- Obtener mayor información internacional sobre los avances logrados en el Control de Calidad de la Auditoría, en lo referente al Control de Calidad Externa efectuado por Auditores independientes, así como mejorar los Controles de Calidad Interna efectuada por las firmas de auditoría u Órganos de Control Gubernamental, en ambos casos proyectados a sus campos de competencia.

2.- La labor especializada de Auditoría para mantener credibilidad y aceptación general en la colectividad debe asegurar que los auditores actúen con honestidad, labor que corresponde incentivarse desde las aulas universitarias a los futuros profesionales en el estricto cumplimiento de sus obligaciones y con el respaldo de una permanente capacitación.

\section{BIBLIOGRAFÍA}

ARTER G., Dennis R.

"Auditorías de calidad para mejorar su comportamiento".

Ediciones Díaz de Santos S. A., España

BASADRE, Jorge

1961. "Historia de la República del Perú" 5a.edición, Tomo I, Lima

BANCOCENTRALDE RESERVA DEL PERÚ 1999. "Tribunal Mayor de Cuentas". En Revista: Moneda 11(113) - feb. - mar.

CASHIN, James A.

1998. "Enciclopedia de la auditoría" 2da. Edición. Oceáno Grupo Editorial, Barcelona

CHÁVEZ ACKERMANN, Pascual

"Fundamentos y principios de contabilidad" Lima.

DÁVILA LADRÓN DE GUEVARA, Carlos 1992."Teoría organizacionales y administración: Enfoque Crítico" Ed. Mc. Graw Hill, Bogotá

ELPERUANO.

Normas publicadas en el Diario Oficial.

Resolución Contraloría No $^{\circ}$ 162-95-CG 1995

Resolución Contraloría Nº 141-99-CG 2511-99

FOWLER NEWTON, Enrique

"Auditoría aplicada" Ediciones Macchi, Argentina

FULMER Robert M.

1981. "Administración moderna" D.F.

México.

GEORGE, Claude

1992. "Historia del pensamiento administrativo" D.F. México, Prentice Hall. 
GROOCOCK, Jhon M.

"La cadena de la calidad"

Ediciones Díaz de Santos S. A.

España

INSTITUTO MEXICANO DECONTADORES PÚBLICOS, A.C.

1996. "Control de calidad en la auditoría de estados financieros".

INSTITUTO MEXICANO DE CONTADORES PÚBLICOS, A.C./FEDERACIÓN DECOLEGIOS PROFESIONISTAS

1997. Normas Internacionales de Auditoría.

JIMENEZCASTRO, Wilburg

1965. "Introducción al estudio de la teoría administrativa" 2a. edición. Fondo de Cultura Económica, D. F. México.

MAS Jordi / RAMIO Carles

1998. La auditoría operativa en la práctica". Alfa Omega Grupo Editor S.A., Colombia.

MEREA LLANOS, Victor Manuel 1994."Análisis de los procedimientos de contabilidad y de la auditoría aplicados en el Perú desde 1535 hasta 1821".Universidad Particular Inca Garcilaso de la Vega (Tesis para optar el grado de Magister en Contabilidad). Lima.

ROSTOROWSKI DIEZCANSECO, María 1995."Historia del Tahuantinsuyo". Instituto de Estudios Peruanos. Lima.

SARV , SINGH, SOIN

1997. "Control de la calidad total". Ediciones Mc Graw Hill, México.

SCHONBERGER, Richard J.

"Manufactura de clase mundial para el próximo siglo". México.

SLOSSE, Carlos A. y otros.

"Auditoría un nuevo enfoque empresarial" Ediciones Macchi, Argentina.
COOPERS \& LYBRAND

"Manual de Auditoría". España.

TZU SUN/MARISCAL, Enrique

1996. "El arte de la guerra: Para nuevos líderes", Obelisco. Barcelona. 